\title{
Zur Gliederung mittel- und jungpleistozäner Schichten in der Niederrheinischen Bucht
}

\author{
Von Karlheinz Kaiser und Rudolf Schütrumpf, Köln
}

Mit 5 Abbildungen und 2 Tabellen im Text

$\mathrm{Zusammenfassung.} \mathrm{Vom} \mathrm{Wasserwerk} \mathrm{Bensberg} \mathrm{östlich} \mathrm{Köln} \mathrm{und} \mathrm{einer} \mathrm{Kiesgrube} \mathrm{bei}$ Efferen westlich Köln werden aus einer Tiefe von 16-18,20 m unter der Oberfläche der Unteren Mittelterrasse des Rheines (ca. $58 \mathrm{~m}$ über NN.) fossilführende Ton- und Feinsandschichten mit humosen Einschaltungen beschrieben, die eine stratigraphische Trennung hangender von liegenden Sand-Kies-Folgen ermöglichen. Sedimentpetrographische Untersuchungen erweisen eine Zuordnung der Liegendserien zur spätmindeleiszeitlichen Mittle re n Mittelter rasse des Rheines, deren Verbreitung im Niederrheingebiet somit nicht nur auf wenig breite Rinnen („Rinnenschotter") beschränkt ist, sondern wie im Mittelrheintal auf der ganzen Talbreite, hier jedoch begraben von den Ablagerungen der Unteren Mittelterrasse, entwickelt sein dürfte. Die palynologische Untersuchung der humosen Einschaltungen in den Ton- und Feinsandschichten am Wasserwerk Bensberg vermittelt eine Florengemeinschaft, die auf Grund hoher Abies-Alnus-Picea-Werte auf ein spätinterglaziales Alter hindeutet und nach den stratigraphischen Gegebenheiten nur den mindel/rißinterglazialen $\mathrm{Kr}$ efelder Schichten zugeordnet werden können.

Die durchgeführten Untersuchungen veranlaßten außerdem eine kritische Überprüfung der derzeitig vorliegenden Gliederungsversuche mittel- und jungpleistozäner Schichtenfolgen in der Niederrheinischen Bucht. Die $\mathrm{Kempener} u n d \mathrm{Neuwerker} \mathrm{Schichten} \mathrm{müssen} \mathrm{mit}$ größter Wahrscheinlichkeit, vor allem nach den stratigraphischen Gegebenheiten und den faunistischen Befunden, von den Krefelder Schichten getrennt und dem Gerdau-Interstadial zugeordnet werden, wobei erstere einem späten und letztere einem mittleren Abschnitt dieses Drenthe/WartheInterstadials zugehören dürften. Die klimazeitliche Einordnung aller entsprechenden Schichtenfolgen der Niederrheinischen Bucht wird in ciner Tabelle darzulegen versucht.

$\mathrm{S} \mathrm{u} \mathrm{m} \mathrm{ma} \mathrm{r} \mathrm{y.} \mathrm{From} \mathrm{the} \mathrm{waterworks} \mathrm{of} \mathrm{Bensberg} \mathrm{east} \mathrm{of} \mathrm{Cologne} \mathrm{and} \mathrm{a} \mathrm{gravel-pit} \mathrm{near} \mathrm{Efferen}$ west of Cologne, there have been described layers of clay and fine-sand, containing fossils with humus layers from a depth of about 16 to 18,20 meters below the surface of the Lower MiddleTerrace of the Rhine (i.e.: about $58 \mathrm{~m}$ above sea-level). These sediments enable us to separate stratigraphically in the sand-gravel-sequences the overlying from the underlying stratum one. Heavy mineral analysis shows, that the underlying series belongs to the $\mathrm{Midd} \mathrm{e} \mathrm{Midd}$ e Ter race of the Rhine (of Late-Mindel-age). The distribution of this terrace within the LowerRhine-Basin thus cannot be limited only to some narrow channels ("Channel-Gravel"), but is supposed to be developped over the whole breadth of the valley, like in the Middle-Rhine-Valley, yet here buried under sediments of the Lower Middle-Terrace. The palynologic investigation of the humus layers within the clay and finesand-layers of the waterworks of Bensberg shows a flora indicating a late-interglacial age by their high percentage of Abies-Alnus-Picea-values. According to the stratigraphical findings they can only be attached to the Mindel/Riß-interglacial K r e f e ld - B ed s.

The investigations carried out led to a critical examination of the existing attempts of differenciating the Middle-Pleistocene beds in the Lower-Rhine Basin. The $\mathrm{K}$ e m p e n a n d $\mathrm{Ne} u$ e $\mathrm{rk}$ $\mathrm{B}$ e d s have to be separated from the Krefeld Beds, according to both the stratigraphic and the floristic and faunistic findings, and must be attached to the Gerdau-Interstadial, the first one probably belonging to a late and the latter (much more probably) to a middle part of this Drenthe/Warthe-Interstadial. The authors try to give a climatic-chronologic table of all Middle Pleistocene beds in the Lower-Rhine-Basin.

Rés u mé. De l'ouvrage hydraulique de Bensberg à l'est de Cologne et d'une couche près d'Efferen à l'ouest de Cologne, les auteurs décrivent des couches d'argile et de sable très fin contenants poches humeuses, qui permettent une séparation stratigraphique des lits inférieurs et supérieurs dans cette séquence de sable et de gravier. La profondeur était 16-28,20 m sous la surface de la Terrasse-Moyenne Inférieure du Rhin (près de $58 \mathrm{~m}$ au delà du niveau de la mer). Des investigations pétrologiques preuvent une connection des séries inférieures à la $\mathrm{T}$ e $\mathrm{r} r$ a s $\mathrm{se-}$ Mo ye n e I n te rmédia i r e du Rhin (Mindel-glaciaire tardif). Ainsi leur distribution ne se limite pas seulement à un canal peu large ("Rinnenschotter"), mais comme dans la vallée du Moyen Rhinelle, est développée à travers tout le talweg: cependant ici enterrée sous les sédiments de la Terrasse-Moyenne Inférieure. Les investigations palynologiques des poches humeuses dans les séries d'argile et de sable très fin ont montré une association des flores indiquant un âge inter- 
glacial tardif par suite des hautes valeurs de Abies-Alnus-Picea. Selon les évidences on les adjoint seulement aux Séries de K refeld (Interglacial Mindel/Riß).

Les investigations éxécutées firent désiderable une rééxamination des essais actuels qui divisent les séries du Pleistocène Moyen dans le Bassin du Rhin-Inférieur. Selon les faits floristiques et faunistiques, les $\mathrm{L}$ its de Kempen et $\mathrm{Neuw}$ erk sont séparés des $\mathrm{L}$ its de Krefeld et adjoints l'Interstadial de Gerdau, les premiers appartenants probablement à une période plus récente et les derniers à la période moyenne de cet Interstadial (Drenthe/Warthe). Par un tableau, les auteurs tentent une classification climato-chronologique de toutes les séries du Pleistocène Moyen au Bassin du Rhin-Inférieü.:

\section{A. Einleitung}

Auf dem Gelände der Wasserwerke Bensberg östlich Köln wurde vor etlichen Jahren im Bereich der Unteren Mittelterrasse (ca. $58 \mathrm{~m}$ über NN.) nördlich der Bruchbachniederung zwischen Brück und Benningsfeld 3 Brunnen angelegt. Dabei ist gleichfalls mit zuvor hier ausgeführten Bohrungen ein Profil der Unteren Mittelterrasse erschlossen worden, das in einer Tiefe von 16-18 m fossilführende Ton- und Feinsandschichten mit humosen Einschaltungen anzeigte und somit eine stratigraphische Trennung hangender von liegenden Sand-Kies-Folgen ermöglichte (Abb. 1).

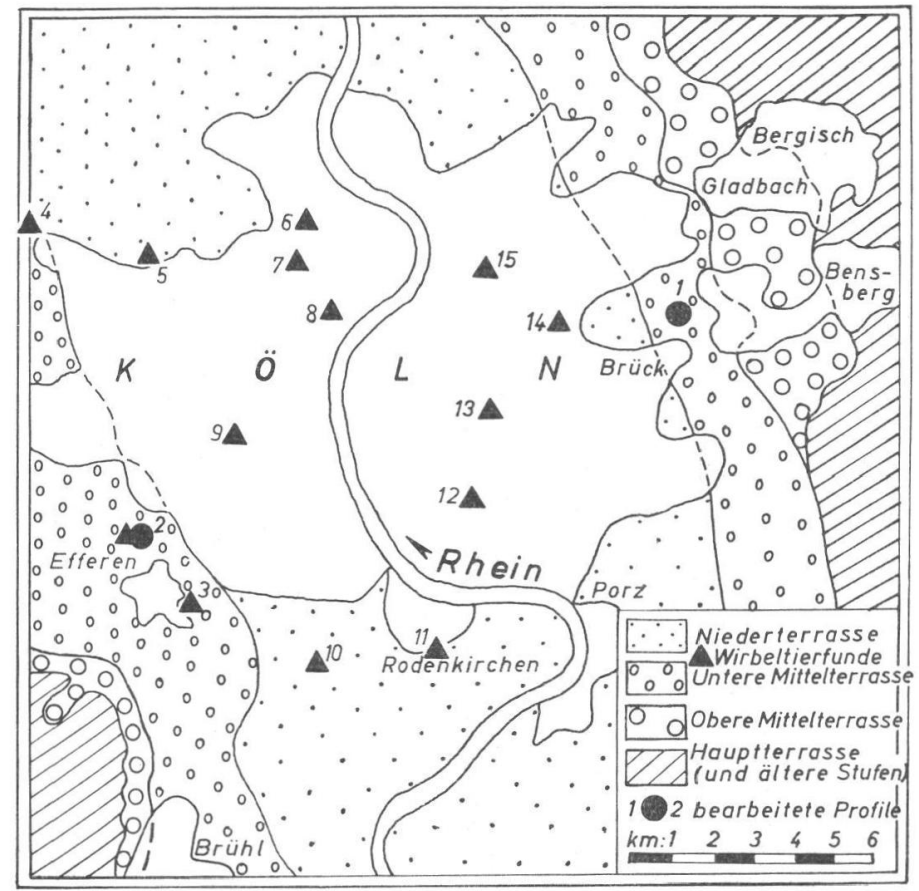

Abb. 1: Fossilfundpunkte in mittel- und jungp'eistozänen Ablagerungen der Kölner Bucht

1: Mindel/Riß-Interglazial am Wasserwerk Bensberg (Untere Mittelterrasse).

2. Kiesgrube bei Efferen (Untere Mittelterrasse): Molare von Elephas (Mammuthus) primigenius

(Blum.) aus der Unteren Mittelterrasse. - Elefanten-Stoßzahn aus der Mittleren Mittelterrasse

(19 $\mathrm{m}$ unter der Geländeoberfläche).

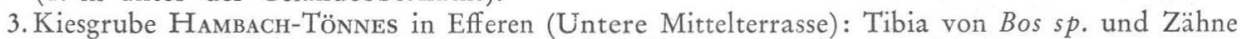
von (wahrscheinlich) Sus scrofa LinN. aus der Unteren Mittelterrasse.

4: Kiesgrube der Kölner Mörtelwerke am Neuenhof bei Köln-Bock!emünd (Untere Mittelterrasse): Molare und Stoßzähne von Elephas (Mammuthus) primigenius (BLum.) aus der Unteren Mittelterrasse.

5: Kiesgrube der Terra-Werke an der Flughafenstraße in Köln-Ossendorf (Niederterrasse): Molare von Elephas (Mammuthus) primigenius (Bцum.) aus der Alteren Niederterrasse. 
6: Kiesgrube Gebr. Schorn am Ginsterpfad in Köln-Weidenpesch (Niederterrasse): Molare, Stoßzahn und Tibia von Elephas (Mammuthus) primigenius (BLum.) aus der Alteren Niederterrasse.

7: Kiesgrube HockfaHrT in Köln-Mauenheim (Niederterrasse): Tibia von Elephas (Mammuthus) primigenius (BLum.) aus der Alteren Niederterrasse.

8: Kiesgrube Gebr. RITZ am Bilderstöckchen in Köln-Nippes (Niederterrasse): Linker Unterkiefer mit Molar eines jugendlichen Exemplares und zahlreiche Mo!are von Elephas (Mammutbus) primigenius (Blum.), rechter Metatarsus von Coelodonta antiquitatis (Blum.) und Knochenfragmente (wahrscheinlich von einem Geweihträger) aus der Alteren Niederterrasse.

9. Baugrube des Wiso-Fakultät-Gebäudes an der Universität in Köln-Lindenthal (Niederterrasse): Oberkieferbruchstück mit 2 Molaren von Bos primigenius BoJ., Knochenfragment (wahrscheinlich) von Equus sp. und Halswirbelsäu'e von Balaena mysticetus L. aus den Basalschichten der bimssteinführenden Jüngeren Niederterrasse (vgl. U. JUX \& K.A. Rosenbauer 1959).

10: Kiesgrube TeHLEN in Rondorf bei Köln (Niederterrasse): Molare und ein Stoßzahn von Elephas (Mammuthus) primigenius (BLum.) aus der Älteren Niederterrasse.

11: Kanalbau-Aufschluß an der Sürther Straße in Köln-Rodenkirchen (Niederterrasse): ElefantenStoßzahn aus der Älteren Niederterrasse.

12: Kiesgrube Nowis \& OTTEN nahe der Autobahn an der Siegburger Straße östlich Köln-Poll Niederterrasse): Linker Femur von Elephas (Mammutbus) primigenius (Blum.) aus der Ailteren Niederterrasse.

13: Kiesgrube Weitmann am Vingster Ring in Köln-Vingst (Niederterrasse): Elefanten Stoßzahn aus der Alteren Niederterrasse.

14: Kiesgrube am Schlagbaum in Köln-Merheim (Niederterrasse): Cerviden-Tibia aus der Alteren Niederterrasse.

15: Kiesgrube WACKER in Köln-Mülheim (Niederterrasse): Humerus von Balaena mysticetus L. aus den Basalschichten (12 m unter der Geländeoberfläche) der bimssteinführenden Jüngeren Niederterrasse (vgl. U. Jux \& K. A. Rosennauer 1959).

Desgleichen war bis 1959 in einer Kiesgrube an der Straße von Köln-Klettenberg nach Stotzheim nordnordwestlich Efferen nahe der Autobahn Köln-Aachen ein Profil der Unteren Mittelterrasse des Rheines (ca. $58 \mathrm{~m}$ über NN.) aufgeschlossen, das hinsichtlich der Abfolge der Schichten dem am Wasserwerk Bensberg sehr ähnlich ist. Auch hier fanden sich unter 1-2 m mächtigen Lößlehmen und Sand-Kies-Folgen in einer Tiefe von 16,50 bis $18,20 \mathrm{~m}$ Feinsande und Tone mit dünnen humosen Einlagerungen, worunter noch ca. $2 \mathrm{~m}$ mächtige Grobkiesbänke (z. T. schon unter dem Grundwasserspiegel) angeschnitten waren (Abb. 1). Die Bergung eines leider nicht näher bestimmbaren Elefantenstoßzahnes im Dezember 1955 aus den liegenden Grobschottern war schließlich der Anlaß einer genaueren Untersuchung des hier aufgeschlossenen Terrassenprofils.

Herzlichen Dank schulden wir Prof. M. Schwarzbach, Köln und Prof. P. Woldstedt, Bonn, die uns wertvolle Hinweise gaben; desgleichen Dozent Dr. U. Jux, z. Zt. Baton Rouge (USA), der uns die Proben vom Wasserwerk Bensberg vermittelte und mit seinem Rat die Untersuchungen förderte. Ferner gebührt unser Dank Dr. A. Prashnowsky, Würzburg, und Dr. R. Vinken, Hannover, die die Schwermineral-Aalysen durchführten. Nicht zuletzt sei aber auch jenen Herren vom Wasserwerk Bensberg und dem derzeitigen Besitzer der Kiesgrube Efferen gedankt, die uns schließlich die Möglichkeit zur Untersuchung beider Profile gaben.

\section{B. Zur Gliederung mittelpleistozäner Terrassensedimente im Kölner Raume}

\section{Das Mindel-Riß-Interglazial und die hangenden und liegenden Sand-Kies-Folgen am Wasserwerk Bensberg bei Köln}

a) Die Zusammensetzung, Herkunft und zeitliche Stellung der Terrassensedimente

Die Zusammensetzung der hangenden und liegenden Sand-Kies-Folgen am Wasserwerk Bensberg (Abb.1) zeigt ein für die Untere und Mittlere Mittelterrasse des Rheines in der Niederrheinischen Bucht typisches Bild (Abb. 2): 40-50\% Quarz, 2-3\% Lydit, 20-30\% Quarzit, 15-20\% Sandsteine und Grauwacken, 8-15\% Tonschiefer, 2-30\% 


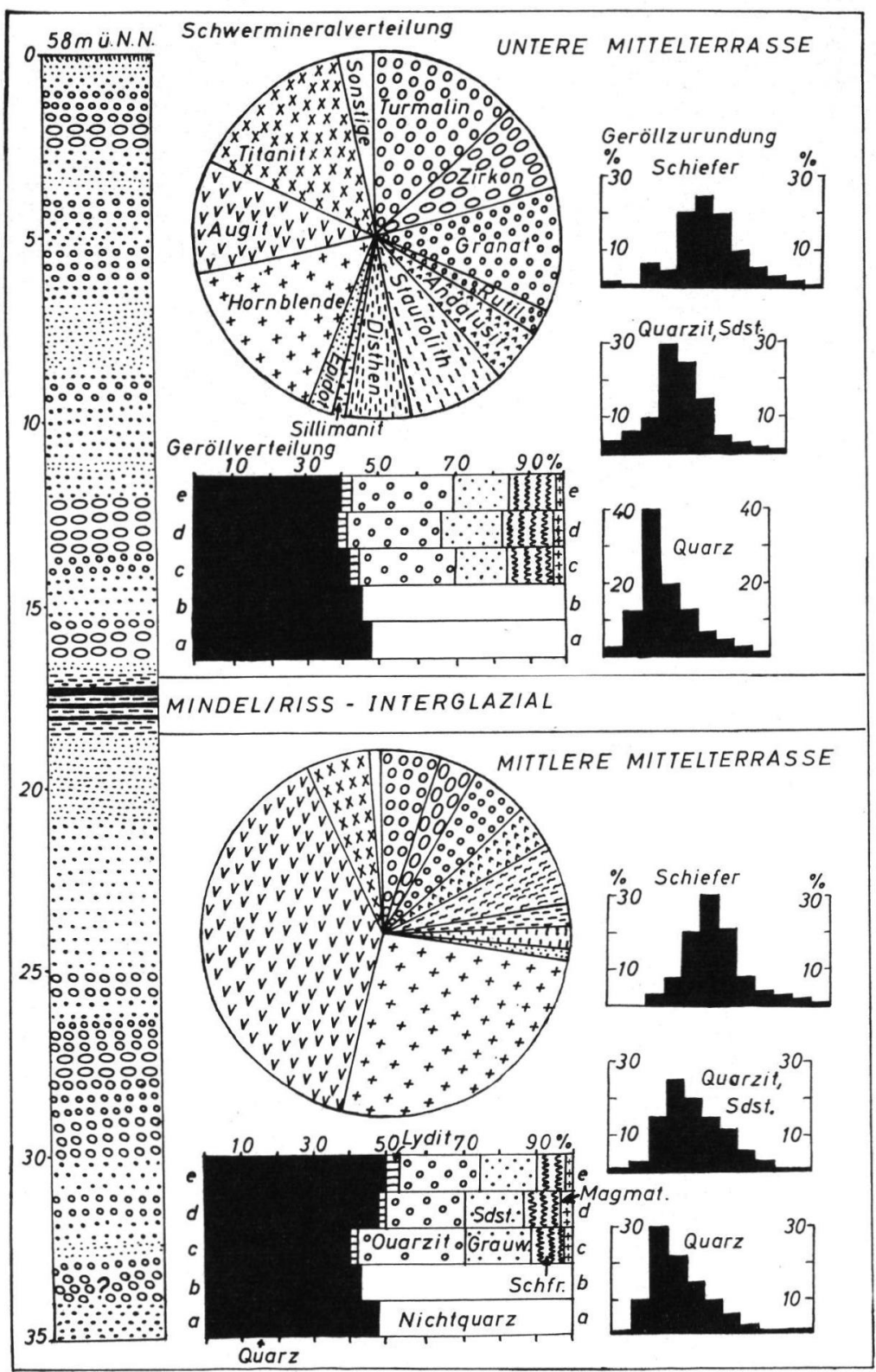

Abb. 2. Die mittelpleistozänen Schichtenserien vom Wasserwerk Bensberg östlich Kö!n.

magmatische und sonstige Geröllkomponenten. Leitgerölle des Rheines und seiner mitielund oberrheinischen Einzüge sind häufig zu finden, so daß kein Zweifel besteht, die Terrassensedimente als Rheinablagerungen aufzufassen, zumal sich eine nennenswerte Beteiligung von Siegmaterial, die man vielleicht hier noch erwarten könnte, in den Diagrammen der Geröllverteilung hätte abzeichnen müssen (vgl. K. KAISER 1956). Das Verhältnis Quarz/Buntschotter ist in den liegenden Serien nur unwesentlich höher (im Durchschnitt sind hier nur 2\% Quarz mehr enthalten), woraus also keine weiteren Schlüsse hinsichtlich 
der Zuordnung der liegenden und hangenden Sand-Kies-Folgen hergeleitet werden können.

In der Schwermineralverteilung $(\mathrm{Abb} .2)^{1}$ ) zeichnen sich hinsichtlich der hangenden und liegenden Schotter größere Unterschiede ab. So sind im Durchschnitt in den Jiegenden Serien über 70\% Hornblende, Augit und Titanit (davon ca. 45\% Augit und Titanit) vertreten, denen in den hangenden Serien nur ca. 40\% an Hornblende, Augit und Titanit (davon ca. 25\% Augit und Titanit) gegenüberstehen. Die ersteren zeigen demnach eine für den „Rinnenschotter“, letztere für die Untere Mittelterrasse des Rheines in der Niederrheinischen Bucht typische Verteilung der Schwerminerale (vgl. u. a. J. I. S. ZonneVELD 1956).

Die Durchschnitts-Zurundungs-Morphogramme (vg1. K. Karser 1956) der liegenden und hangenden Schotter zeigen weitgehende Übereinstimmung (Abb. 2) und weisen auf glaziale Klimabedingungen zur Zeit ihres Transportes und ihrer Sedimentation hin (äußerst mäßige Geröllzurundung). Daß dabei die Quarze schlechter als Quarzite, Sandsteine und Grauwacken, und diese wiederum weniger stark als die Tonschiefer gerundet sind, entspricht durchaus den allgemeinen Abrollungsbedingungen, die sich ja weitestgehend, abgesehen von Transportweite und den klimatischen Gegebenheiten, nach der Gesteinsart richtet (vgl. u. a. K. KaISER 1956).

Während für die Untere Mittelterrasse auf Grund der Möglichkeit ihrer Verknüpfung mit den rißhochstadialen Eisrandbildungen am Niederrhein (vgl. u. a. K. KaISER 1957) und ihrer Unterlagerung durch fossilführende sandig-tonige Schichten (Mindel/Riß-Interglazial) eine kaltzeitliche Stellung (Drenthe-Stadium) angezeigt ist, so dürfte andererseits auf Grund oben aufgezeigter geröllmorphometrischer Befunde die Sedimentation der „Rinnenschotter" entgegen der Ansicht von H. Karrenberg (1951)2) und H. W. Quitzow (1956), der diese in die erste Hälfte des Mindel/Riß-Interglazials stellte, in die ausgehende Mindel-Eiszeit zu datieren sein. Zudem hat R. Teichmülleer (in: G. von Der Brelie, K. Kilpper \& R. TeichmüLleR 1960) erstmalig am Niederrhein aus Frimmersdorf an der Erft sowohl aus den Ablagerungen der Unteren Mittelterrasse als auch aus denen des „Rinnenschotters" synchrone Eiskeile und Kryoturbationen beschrieben, die also die sicher kaltzeitliche Stellung beider Terrassenschotter wahrscheinlich machen.

\section{b) Das Mindel/Riß-Interglazial}

$\mathrm{Da}$ beim Besuch der Fundstelle die Bohrungen und Brunnenbauten seit längerer Zeit abgeschlossen, die Bohrlöcher verrohrt und die Brunnen bereits ausgekleidet waren, stan-

1) Die Schwermineral-Analysen wurden durch Dr. A. Prashnowsky im Geologischen Institut der Universität Köln durchgeführt. Bei beiden Diagrammen wird die durchschnittliche Schwermineral-Verteilung angezeigt, indem eine Serie horizontierter Proben sowohl aus den liegenden als auch aus den hangenden Sand-Kies-Folgen gut vermischt wurden. Aus den Liegendserien wurden 254, aus den Hangendserien $3 \$ 1$ Mineralkörner ausgezählt. Leider sind Alterit und Saussurit nicht berücksichtigt worden, so daß die gewonnene Darstellung etwas von den Normalbildern der Schwermineral-Verteilung der Unteren und Mittleren Mittelterrasse („Rinnenschotter") des Rheines abweichen muß. Auch sind die verschiedenen Hornblenden (besonders wäre Basaltische Hornblende wichtig) nicht gesondert ausgezählt worden. In den Liegendserien waren alle Mineralkörner meist eckig ausgebildet, während in den Hangendserien 'Turmalin, Zirkon, Rutil, Granat, Staurolith und auch Titanit z. T., auf Umlagerungen hinweisend, stärker gerundet und hier nur Hornbiende und besonders Augist meist eckig erschienen.

2) H. KARRENBERG (1951): „Eine ähnliche Zweiteilung der Mittelterrasse ist im Gebiet westlich Bensberg und im südlich anschließenden Königsforst festgestellt worden. Hier ist offenbar an einer Randstaffel des Rheintalgrabens die Hauptterrasse versenkt und wird durch ein Interglazial von Krefelder Charakter und Mittelterrassenablagerungen überlagert."

Erstmalig wird hier also über jenes Interglazial, von dem nachfolgend die Rede ist und das diese "Zweiteilung der Mittelterrasse" bedingt, auf Grund vorliegender Bohrergebnisse berichtet; außer den zitierten sind keine weiteren Mitteilungen darüber gemacht worden. H. W. QuiTzow (1956) hat die dort "tektonisch abgesenkten Hauptterrassenschotter" als (ungestört lagernde) "Rinnenschotter" des Rheines umgedeutet und deren auf Grund der Bohrergebnisse angezeigte Verbreitung auf seiner Übersichtskarte verzeichnet. 
Tabelle 1

Pollen-Zusammensetzung des Mindel/Riß-Interglazials vom Wasserwerk Bensberg bei Köln.

\begin{tabular}{|c|c|c|c|c|c|c|c|c|c|c|c|c|c|c|c|c|c|c|c|c|}
\hline \multirow[b]{2}{*}{ 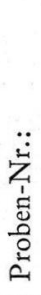 } & \multirow[b]{2}{*}{$\underset{\ddot{v}}{\ddot{\sim}}$} & \multirow[b]{2}{*}{ Schichtenart: } & \multicolumn{12}{|c|}{ Ba ampollen: } & \multicolumn{5}{|c|}{ Nichtbaumpollen: } & \multirow{2}{*}{$\begin{array}{c}\text { Spor.: } \\
\\
\ddot{\ddot{\Xi}} \\
\stackrel{\vec{\pi}}{\pi}\end{array}$} \\
\hline & & & $\frac{\ddot{3}}{\tilde{a}}$ & $\ddot{\ddot{\Sigma}}$ & 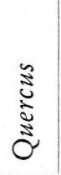 & $\frac{i \sqrt{i}}{\ddot{i}}$ & 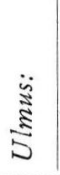 & 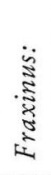 & $\frac{\ddot{\Xi}}{\ddot{z}}$ & $\sum_{i=1}^{\ddot{z}}$ & $\frac{\ddot{3}}{\grave{\Xi}}$ & $\ddot{\ddot{z}}$ & $\begin{array}{l}\ddot{\ddot{\Xi}} \\
\ddot{\tilde{\nabla}}\end{array}$ & 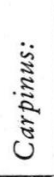 & $\frac{0}{3}$ & 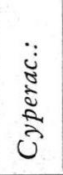 & 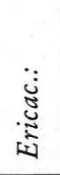 & $\begin{array}{l}\ddot{\Xi} \\
\tilde{\delta} \\
o \\
o \\
\tilde{\Xi} \\
\tilde{U}\end{array}$ & $\frac{\rho^{8}}{\ddot{8}}$ & \\
\hline 1 & $\begin{array}{l}16.10 \mathrm{~m} \\
\mathrm{bis} \\
16,60 \mathrm{~m}\end{array}$ & $\begin{array}{l}\text { stark humoser, schwarz-brauner } \\
\text { Ton im gelben Lehm }\end{array}$ & 2,5 & 5,5 & 0,5 & - & 0,5 & 1 & 30 & 2 & 0,5 & 6 & 52,5 & 1 & 0,5 & - & - & - & 0,5 & 0,5 \\
\hline 2 & $\begin{array}{l}16,60 \mathrm{~m} \\
\text { bis } \\
17,40 \mathrm{~m}\end{array}$ & $\begin{array}{l}\text { schwarzer Torf } \\
\text { (cf. Bruchwald) }\end{array}$ & - & 7 & 2 & - & - & - & 34 & 2 & 3 & 15 & 38 & 1 & 1 & 1 & - & - & 2 & 3 \\
\hline 3 & $?$ & feingeschichtete Gyttja & 2,5 & 9,5 & 4,5 & - & 1 & 2 & 26 & 7,5 & 2 & 7,5 & 45,5 & - & 1 & 3 & 1 & - & 1 & 13 \\
\hline 4 & $?$ & feingeschichtete Gyttja & 1 & 5,5 & 4 & 0,5 & 0,5 & - & 36 & 5 & 5 & 13,5 & 34 & - & 1,5 & 1,5 & 0,5 & - & 1 & 1 \\
\hline 5 & $?$ & $\begin{array}{l}\text { feingeschichtete Gyttja } \\
\text { (gröber als Nr. } 3 \text { u. 4) }\end{array}$ & 4 & 14 & 2 & 1 & 1 & - & 38 & 4 & 3 & 3 & 34 & - & - & 3 & 2 & 3 & 1 & 57 \\
\hline 6 & $?$ & $\begin{array}{l}\text { geschichtete Gyttja mit Schicht- } \\
\text { grenze zum blau-grauen, } \\
\text { nicht humosen Ton }\end{array}$ & - & 8 & 12 & - & 1 & + & 32,5 & 13 & 2 & 8 & 35,5 & 1 & 2 & 10 & - & - & 1 & 10 \\
\hline
\end{tabular}




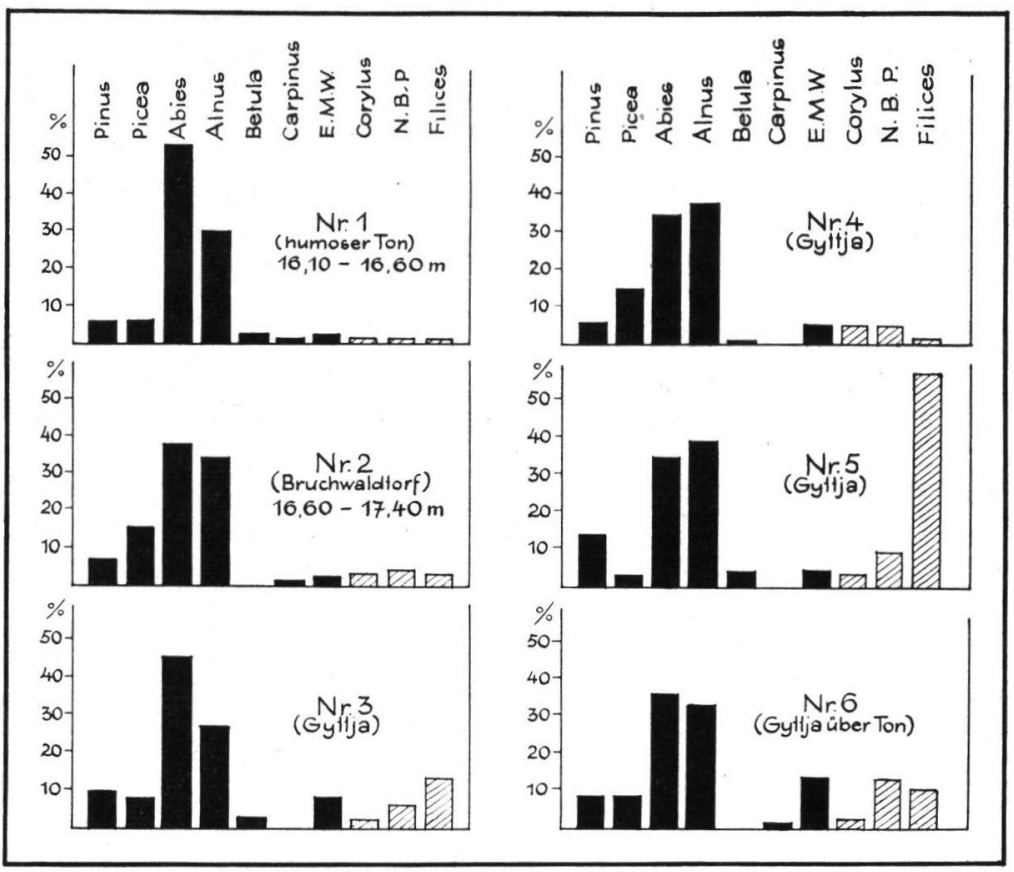

Abb. 3. Pollen-Diagramme des Mindel/Riß-Interglazials vom Wasserwerk Bensberg.

den zunächst nur 2 Proben zur Verfügung, die (in Einmachgläsern verpackt) im Wasserwerk Bensberg und im Geologischen Institut Köln aufbewahrt worden waren. Doch leider sind auch diese nur beschränkt auswertbar, da es sich um Durchschnittsproben aus 50 bzw. $80 \mathrm{~cm}$ Schichtenmächtigkeit handelt, und zwar:

Probe Nr. 1: 16,10-16,60 m (bezeichnet als „Schluff, braungrau-schwarz; Ton/ Braunkohle").

Es handelt sich um einen stark eisenhaltigen gelben Lehm, etwas tonig, mit humosen Einlagerungen. Die analysierte Probe bestand aus braunschwarzem, stark humosem Ton von nahezu torfigem Charakter.

Probe Nr. 2: 16,20-17,40 m (bezeichnet als "Braunkohle, schwarz mit Holz“).

Es handelt sich um einen \pm stark zersetzten Bruchwaldtorf.

Zusätzlich wurden auf der Halde der ehemaligen Brunnenbaustelle 3 eine Anzahl von Handstücken aufgesammelt, die auf.Grund der zu beobachtenden Schichtung noch in situ vorlagen und orientiert werden konnten. Auch diese wurden pollenanalytisch untersucht.

Insgesamt wurden von obigem Material 10 Analysen durchgeführt, davon wegen des $z$. T. geringen Pollengehaltes nur 6 quantitativ. Die Aufbereitung der Proben erfolgte mit. Kalilauge und Natriumperborat (nach M. vaN CAMPo 1950). Die Analysenergebnisse sind in Tabelle 1 und Abb. 3 zusammengestellt worden.

Kennzeichnend für alle Spektren ist die Vorherrschaft der Nadelhölzer (besonders von Abies, die maximal mit 53\% erscheint) bei hohen Alnus-Prozenten (26,5-39\%). Die wärmeliebenden Arten, die Komponenten des Eichenmischwaldes (E.M.W.) und die Hasel, spielen nur eine untergeordnete Rolle.

Im nord- und nordwesteuropäischen Raum weist ein solch hoher Anteil von Tanne (und Fichte) zumeist auf interglaziales Alter hin. Vergleicht man die ermittelten Pollenspektren mit bekannten, stratigraphisch gesicherten Interglazial-Profilen und Einzel- 
proben aus Nordwest-Europa, so ergibt sich, daß die aus dem letzten Interglazial (Riß/ Würm- oder Eem-Interglazial) stammenden Ablagerungen zwar ebenfalls eine kurze Tannen-Phase aufweisen, sofern die Probenfolge dicht genug gewählt ist, daß die AbiesMaxima hierin aber nur selten Werte von $10 \%$ übersteigen. Anders dagegen liegen die Verhältnisse im Mindel/Riß-Interglazial (und "Ohe-Interglazial“). Hier werden in den entsprechenden Ablagerungen hohe Tannen-Werte (größtenteils in der gleichen Größenordnung wie in unseren in Frage stehenden Spektren) erreicht .Auch hohe Alnus-Werte daneben sind für dieses Holstein-Interglazial typisch.

Am besten lassen sich die Spektren mit jenen aus den Krefelder Schichten der niederrheinischen Stauchwälle vergleichen, auf deren Altersstellung zuvor schon eingegangen wurde. Demnach können nach dem derzeitigen Stand unserer Kenntnis die humosen Schichten der Unteren Mittelterrasse am Wasserwerk Bensberg bei Köln ebenfalls in das Mindel/Riß-Interglazial datiert werden.

Allerdings umfassen die ermittelten Spektren (auch die nicht quantitativ analysierten) nur einen kürzeren Abschnitt dieses Interglazials, nämlich eine fortgeschrittene und etwas kühlere Periode mit Dominanz der Koniferen nach dem temperierten Abschnitt der Warmzeit. Die Probe Nr. 6 dürfte unter den untersuchten die älteste sein, nachdem sich auch in dem betreffenden Handstück eine scharfe Grenze zwischen nicht humosem, blaugrauem Ton und gut geschichteter Grobdetritus-Gyttja mit torfigem Habitus abzeichnete. So würden auch die relativ hohen Eichen-Werte von $12 \%$ in dieser einen Probe eine Erklärung finden, indem sie auf den Übergang zum vorhergehenden laubholzreichen, wärmeren Abschnitt hinweisen. Auch die gesamten Spektren der Krefelder Schichten am Niederrhein zeigen ja nur die letzte Phase des betreffenden Interglazials mit Dominanz der Koniferen an.

\section{Das Schichten-Profil der Mittelterrasse von Efferen bei Köln}

In ihrer Zusammensetzung sind auch die Mittelterrassenkiese von Efferen durch typisch rheinische Gerölle bestimmt. Es wurden insgesamt 20 horizontierte Proben hinsichtlich der Geröll- und Schwermineralverteilung sowie geröllmorphometrisch untersucht, davon 3 aus den antonigen Feinsandschichten und eine aus deren liegenden Grobkiesbänken. Das Quarz/Buntschotter-Verhältnis ist äußerst großen Schwankungen unterworfen (Quarzgehalt zwischen 15 und 60\%), doch zeigen die Durchschnittsdiagramme (Abb. 4) eine für die Untere (Hangendserien) und Mittlere Mittelterrasse (Liegendserien) des Rheines in der Niederrheinischen Bucht typische Geröllzusammensetzung. Allerdings ist hier der Quarzgehalt im Durchschnitt um ca. 5\% geringer als in den entsprechenden Ablagerungen am Wasserwerk Bensberg. In den Liegendserien ist der Quarzgehalt um ca. 5\% höher als in den Hangendserien. $\mathrm{Da}$ jedoch aus den Grobkiesen unter den antonigen Feinsanden nur eine Probe zur Verfügung stand, sind daraus zu folgernde Schlüsse zunächst nicht angebracht.

Größere Unterschiede gegenüber den entsprechenden Schichtenserien vom Wasserwerk Bensberg zeigen sich hinsichtlich der Schwermineralverteilung (Abb. 4) ${ }^{3}$ ). Zirkon, Turmalin und Rutil zusammen sind in allen Proben mit weniger als 10\% vertreten (Hangendserien

3) Die Schwermineral-Analysen wurden durch Dr. R. VINKEN im geologischen Institut der Universität Bonn durchgeführt. Insgesamt wurden 20 horizontierte Proben untersucht, wovon nur eine den Liegendserien und 3 den Ton- und Feinsandschichten entstammen. Über die Anzah! der ausgezählten Körner liegen keine genaueren Angaben vor, ebenfalls über die Form der Mineralkörner. Alterit und Saussurit wurden (jedoch nicht gesondert) mit ausgezählt.

Die Schwermineral-Diagramme zeigen innerhalb der Hangendserien bestimmte Schwankungen in Abhängigkeit von der Körnung der Sedimente, denen die jeweiligen Proben entstammen. So ist angezeigt, daß in den Terrassenkiesen die stabilen Minerale (besonders aber Granat) weit stärker angereichert sind als in den feinkörnigeren Terrassensanden; eine Tatsache, auf die wiederholt hingewiesen wurde (vgl, u. a. K. KAISER 1956). 


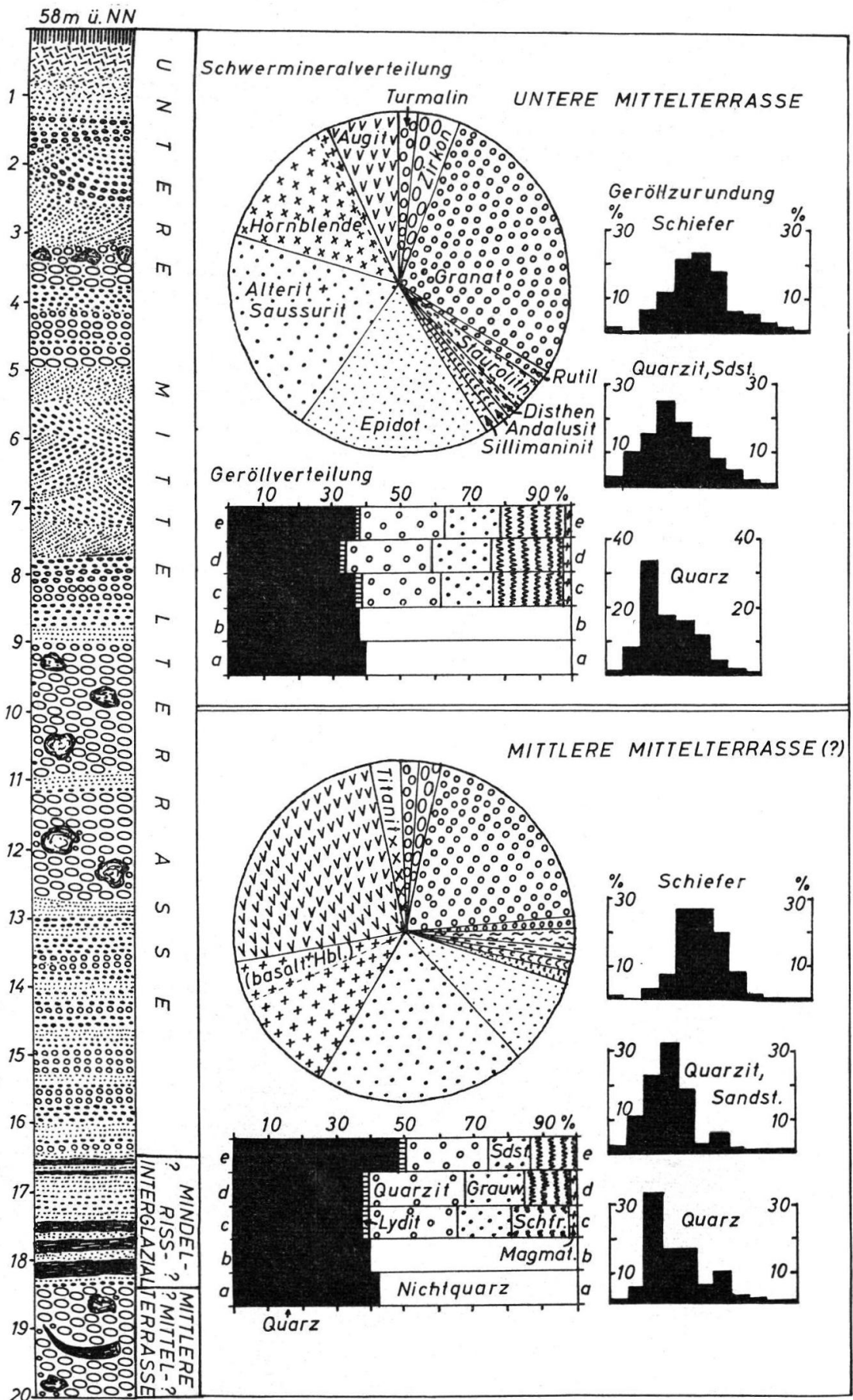

Abb. 4. Die mittelpleistozänen Schichtenserien von Efferen westlich Köln. 
vom Wasserwerk Bensberg: ca. 25\%, Liegendserien vom Wasserwerk Bensberg: ca. 10\% Granat kommt umgekehrt hier mit wesentlich höheren Werten $(20-40 \%)$ vor als am Wasserwerk Bensberg (unter 10\%). Gering ist wieder die Beteiligung von "Metamorphen“ (unter 5\% Hangendserien am Wasserwerk Bensberg: ca. 20\%, Liegendserien am Wasserwerk Bensberg: ca. 10\%). Sehr stark sind wiederum Epidot, Alterit und Saussurit vertreten, die zusammen in allen Proben zwischen 20 und $40 \%$ beteiligt sind. Ein Vergleich zu den Bensberger Proben ist nicht angebracht, da ja dort Alterit und Saussurit nicht ausgezählt wurden; trotzdem zeigen auch dort die geringen Epidot-Werte (unter 5\%) Unterschiede gegenüber dem Profil von Efferen an. Hornblende, Augit und Titanit schließlich sind in Efferen geringer als bei Bensberg vertreten. Zeigen sich im Einzelnen also größere Unterschiede hinsichtlich der Schwermineralverteilung, so ist doch andererseits auch wieder im allgemeinen eine große Übereinstimmung hinsichtlich beider Profile unverkennbar, nämlich der höhere Gehalt an vulkanischen Schwermineralen in den Liegendserien gegenüber den Hangendserien, obschon bei Efferen nicht in demselben hohen Ausmaß wie bei Bensberg ${ }^{4}$ ).

Auch die geröllmorphometrischen Untersuchungen zeigen schließlich ein den am Wasserwerk Bensberg gewonnenen Ergebnissen vollkommen übereinstimmendes Bild. Es soll ferner nicht unerwähnt bleiben, daß in der genannten Kiesgrube bei Efferen verschiedentlich aus den Hangendserien Molare und andere Reste von Elephas (Mammutbus) primigenius (BLum.) geborgen werden konnten. Der Fund eines Stoßzahnes eines leider nicht näher bestimmbaren Elefanten in den Liegendserien im Dezember 1955 war zudem der Anlaß dazu, dieses Kiesgrubenprofil genauer zu untersuchen ${ }^{5}$ ).

Die antonigen Feinsande und besonders die humosen Einschaltungen darin sind von uns wiederholt, jedoch leider ergebnislos auf ihre Pollenführung (es fanden sich nur vereinzelt und zumeist stark korrodierte Pollen) untersucht worden. Konnte hier also nicht

4) Das erstmalige Auftreten vulkanischer Schwerminerale (besonders einer bestimmten Braunen Hornblende) in den Oberen Mittelterrassen des Rheines und die auffällige Anhäufung besonders von Augit in der Mittleren Mittelterrasse des Rheines ("Rinnenschotter") ist seither immer von allen Bearbeitern (vgl. u. a. J. I. S. ZonNeveld 1956, 1958) auf alt- und mittelpleistozäne Vulkanausbrüche in der Eifel zurückgeführt worden. Nachdem vor allem J. FrECHEN (u. a. in: M. Hopmann, J. Frechen \& G. Knetsch 1958) den „altp'eistozänen“ Vulkanismus der Eifel in seiner Verbreitung aufgezeigt und als wenig bedeutend hingestellt hat, scheint es angebracht, die ersten Vorkommen vulkanischer Minerale unter den Sanden der Oberen Mittelterrassen und besonders ihr gehäuftes Auftreten in der Mittleren Mittelterrasse („Rinnenschotter") nicht auf gleichzeitig oder unmittelbar zuvor erfolgte Eruptionen von Vulkanen der Eifel (z. B. Maareifel zwischen Daun, Manderscheid und Bad Bertrich; Rodderberg; Laacher-See-Gebiet) zurückzuführen, sondern mit einer verstärkten Tiefenerosion des Rheines nach der Hauptterrassenzeit, besonders aber nach jener Zeit der Tiefenerosion, die das äußerst stark eingetiefte Bett der Mittleren Mittelterrasse des Rheines schuf, in Verbindung zu bringen; wobei also in allen Teilen des Rheinischen Schiefergebirges erst weite Flächen mit den tertiären vulkanischen Ablagerungen vom Rheinstrom und seinen Tributären angeschnitten und $z$. 'T'. auch ausgeräumt werden konnten (z. B. Westerwald oder Hocheifel). Wolle man das Auftreten vulkanischer Minerale in Terrassensedimenten mit gleichzeitig oder unmittelbar zuvor erfolgten Vulkanausbrüchen in Verbindung bringen, so müßte das für das Rheinstromgebiet gerade in der Niederterrasse angezeigt sein, da ja im Rheinland (besonders in der Eifel) die stärksten quartären Vulkanausbrüche in die Würmeiszeit und ganz besonders in einem späten Abschnitt derselben stattfanden. Das ist jedoch nicht der Fall, da sich die Sedimente der Unteren und Krefelder Mittelterrasse, als auch der Niederterrasse kaum hinsichtlich der Schwermineral-Verteilung unterscheiden lassen, vor allem auch auf Grund der Beteiligung vulkanischer Minerale. Trotzdem könnte hier erst eine weiträumig auf das Rheinische Schiefergebirge ausgedehnte und terrassenzeitlich gut gesonderte Untersuchung der Schwermineralverteilung letzte Klarheit schaffen.

5) Auf Abb. 1 sind die dem Geol. Inst. der Universität Köln aus dem Bereich der Mittel- und Niederterrassen des Rheines im Kölner Raum mitgeteilten Funde von Säugetierresten verzeichnet worden. Es handelt sich hierbei jedoch nur um den Bruchteil des wirklichen Fundmaterials. Ausnahmslos gehören seither alle geborgenen Reste aus diesen Sedimenten einer kaltzeitlichen (Primigenius-) Fauna an. 
wie in Bensberg der Nachweis eines den Sand-Kies-Folgen zwischengeschalteten Interglazials von Mindei/Riß-Charakter erbracht werden, so ist doch auf Grund stratigraphischer und sedimentpetrographischer (besonders der mit Hilfe der Schwermineralanalyse gewonnenen Ergebnisse) Untersuchungsbefunde hier wie dort eine Zweiteilung der Terrassensedimente zwingend, wobei an beiden Stellen die Liegendserien der Mittleren und die Hangendserien der Unteren Mittelterrasse des Rheines zuzuordnen sind.

Aus dem Vorkommen des „Rinnenschotters“ zu beiden Seiten des heutigen Rheintales innerhalb der Kölner Bucht ist schließlich der Schluß zu ziehen, daß der „Rinnenschotter" mit größter Wahrscheinlichkeit nicht nur in besonderen und wenig breiten "Rinnen “ des Mittleren Mittelterrassen-Rheinbettes innerhalb der Niederrheinischen Bucht, wie es H. W. Quitzow (1956) annahm und auf seiner Rheinterrassen-Übersichtskarte verzeichnete, zur Ablagerung kam, sondern wie im Mittelrheingebiet (vgl. u. a. P. Woldstedt 1956), eingesenkt in das System der Oberen Mittelterrassen, auf der gesamten Talbreite. $\mathrm{Da}$ diese Mittlere Mittelterrasse im Mittelrheingebiet auch morphologisch in Erscheinung tritt (z. B. als Apollinaris-Terrasse bei Remagen), so muß am Rande der Köln-Bonner Bucht gegen das Rheinische Schiefergebirge eine Terrassenkreuzung von Unterer und Mittlerer Mittelterrasse vorliegen, wie das ja auch dort für Kieseloolitherrasse (Höhenterrassen), Altere und Jüngere Hauptterrasse einerseits und dann, weit schwächer, auch für Altere und Jüngere Niederterrasse andererseits nachzuweisen ist. Als Ursache der Terrassenkreuzung müssen mit größter Wahrscheinlichkeit tektonische Verstellungen innerhalb der Niederrheinischen Bucht während eines Mindel-Interstadials (vgl. K. KAISER 1956, 1957, wo diese Bewegungen ins Mindel/Riß-Interglazial gestellt werden) angesehen werden. Nach dem zuvor Dargelegten erscheint es aber auch angebracht die Ersetzung des Begriffs „Rinnenschotter“ durch „Ablagerungen der Mittleren Mittelterrasse des Rheines " für das Niederrheingebiet vorzuschlagen, da zu vermuten ist, daß sich die Mittlere Mittelterrasse des Mittelrheintales in ihrer ganzen Breite in die Niederrheinische Bucht, hier dann begraben von den Ablagerungen der Unteren Mittelterrasse, fortsetzt.

\section{Verbreitung, Fauna und Flora der Krefelder, Kempener und Neuwerker Schichten und ihre klimazeitliche Stellung unter den mittelpleistozänen Terrassensedimenten und Eisrandbildungen in der Niederrheinischen Bucht}

In den rißhochglazialen (Drenthe-Stadium) Eisrandbildungen (Stauchwällen) der Niederrheinischen Bucht zwischen Krefeld und Kleve (Abb. 5 ${ }^{6}$ ) sind seit langem eingestauchte Schollen von fossilführenden Ton- und Feinsandschichten bekannt (P. G. KRAUsE 1909, G. Fliegel \& J. Stoller 1910). Sie wurden zunächst den Tegelen-Schichten (i.w.S.)

6) Im Gegensatz zu den Niederlanden hat das Nordische Inlandeis im Raume der Niederrheinischen Bucht während des Drenthe-Stadiums nur 2 Eisrandlagen hinterlassen, die als Krefeider und Mintarder Eisrandlage (K. Kaiser 1957) bezeichnet wurden (Abb. 5). K. N. ThOme $(1958,1960)$ lehnt allerdings eine Verknüpfung der Stauchwälle westlich des Ruhrtales zwischen Kettwig und Duisburg und den inneren Stauchwällen am linken Niederrhein ab (Mintarder Eisrandlage), da er zu dieser Zeit einen nochmaligen Vorstoß des Eises in das Tal der unteren Ruhr bis Kettwig für unwahrscheinlich hält. Er ordnet deshalb die Eisrandbildungen an der Ruhr dem äußersten Eisvorstoß in die Niederrheinische Bucht zu (Krefelder Eisrandlage), die dann kurzfristig überfahren worden seien. Es besteht jedoch kein Zweifel, daß diese Stauchwälle eine geschlossene Eisrandlage darstellen. Die Intensität der Stauchungen ist denen am linken Niederrhein ebenbürtig, und Spuren einer Überformung durch überfahrenes Eis lassen sich nirgends nachweisen, so daß sie sich zweifellos als jünger als der äußerste Eisvorstoß erweisen. Die westwärts gerichteten Entwässerungsrinnen im Hauptterrassensporn zwischen Ratingen und Duisburg hat $K$. $N$. Tноме (1960) als Überlaufrinnen zeitweilig gestauter Ruhrwässer erklärt. Doch scheint auch hier eine Deutung als periglaziale Entwässerungsrinnen z. Zt. der Mintarder Eisrandlage auf Grund ihres Formencharakters ("Trompetentälchen") und ihrer Ursprungslagen in wechselnden Höhenniveaus zwingender (vgl. u. a. K. KAISER 1957). 
gleichgestellt $\left.{ }^{7}\right)$. K. JEssEN $(1924)^{8}$ ) hat dann wohl erstmalig diese Schichten palynologisch untersucht, wobei der hohe Gehalt an Abies-Pollen besonders herausstach, und daraufhin den interglazialen Charakter derselben herausgestellt. Auf Grund stratigraphischer Befunde und der pollenanalytischen. Untersuchungsergebnisse K. JesSEN's, die also eindeutig floristische Unterschiede gegenüber den 'Tegelen-Schichten (i.e.S.) aufzeigten, führte A. Steeger (K. Bertsch \& A. SteEger 1927) die Abtrennung dieser eingestauchten fossilführenden Ablagerungen in den niederrheinischen Stauchwällen, wofür er den Namen Krefelder Schichten prägte, von den eigentlichen Tegelen-Schichten durch. Seitdem sind diese Krefelder Schichten wiederholt und vielerorts in den Stauchwällen (Abb.5) palynologisch und paläozoologisch untersucht und gleich dem "Needien" der Niederlande dem Mindel/Riß-Interglazial zugeordnet worden ${ }^{9}$ ).

Waren also zunächst diese Interglazialbildungen im Niederrheingebiet auf sekundärer Lagerstätte bekannt gemacht worden ${ }^{10}$ ), so wurden seit 1925 auch ähnliche fossilführenden Schichten durch Bohrungen im Bereich der Krefelder Mittelterrasse und „Mörser Niederterrasse" erschlossen. Erstmalig hat K. Bertsch (K. Bertsch, A. Steeger \& U. Steusloff 1931) aus dem Bereich der Krefelder Mittelterrasse, bei Heideck ${ }^{11}$ ) südlich Krefeld und Neuwerk bei Mönchen Gladbach, solche tonigen Ablagerungen palynologisch untersucht. Diese Untersuchung ergab für Heideck eine deutliche Übereinstimmung mit 'dem floristischen Befund der Krefelder Schichten (Abies-Werte 5-30\%). Andererseits ist das Pollenbild von Neuwerk grundverschieden von den anderen ( $\mathrm{H}$ a sel-Werte bis über $70 \%$, Eichenmischwald-Werte bis über $15 \%$, Buchen-Werte bis $20 \%$, keine Tannen-Pollen). Auch die später von K. Jessen (1934, vgl. A. Steeger 1952) vom Groß-

7) Die Tegelen-Schichten wurden erstmalig durch E. Dubors (1905) aus dem deutsch-holländischen Grenzgebiet östlich der Maasniederung zwischen Roermond und Venlo beschrieben und ihre zeitliche Gleichstellung mit dem „Cromer-Forest-Bed“, das ja heute dem Günz/Mindel-Interglazial zugeordnet wird, wahrscheinlich gemacht. Wenige Jahre später haben dann C. \& E. M. REID (1907) die Floren dieser Ablagerungen untersucht und auf Grund der zahlreich vorgefundenen Tertiärrelikte ins Jungtertiär gestellt. W. WUNSTORF \& G. FLIEGEL (1910) schließlich ermöglichten eine stratigraphische Trennung eines oberen (Tegelen-Schichten i.e. S.) und unteren Tonlagers (heute als Reuver-Schichten bezeichnet) durch das Auffinden zwischengeschalteter Sand-KiesFolgen, die sie als „Altesten Diluvialschotter" des Rheines bezeichneten. C. \& E. M. REID (1911) ist dann auch der Nachweis der floristischen Trennung beider Tonlager gelungen. Während sich aber in der niederländischen Literatur schon früh der Name Reuver-Schichten für das untere Tonlager einbürgerte, wurde deutscherseits (A. STEEGER 1931) zunächst dieses untere Tonlager als „Altere Tegelen-Schichten“ bezeichnet.

Während also zunächst schon durch E. Dubors (1905) die interglaziale Stellung der TegelenSchichten (i. e. S.) vermutet und später vor allem durch W. WunstorF \& G. FLIEGEL (1910) sowie A. STEEgER $(1914,1925)$ auch stratigraphisch nachzuweisen versucht wurde, haben andererseits besonders immer Paläobotaniker (C. und E. M. Reid, F. Kirchieimer, H. Weyland u. a.) auf Grund der Floren eine Datierung ins Juntertiär vorgenommen. Die Unterschiede der Auffassungen konnten wohl endgültig erst durch das Auffinden von Eiskeilnetzen in hangenden Reuvertonlagen vom Alter der „Altesten Diluvialterrasse“ durch R. Wolters (1950), über deren Echtheit als Periglazialerscheinungen allerdings berechtigte $\mathrm{Zweifel}$ erhoben worden sind (K. KAISER 1956, 1958), zugunsten einer altinterglazialen Stellung (Donau/Günz-Interglazial) behoben werden.

8) Briefl. Mitteilung an A. Steeger (vgl. A. SteEger 1952).

9) K. Jessen (1924, vg1. A. Steeger 1952) untersuchte Proben vom Oermter Berg (51\% Abies) und Eyeller Berg (bis 43\% Abies), R. PotoniÉ (in: E. Zimmermann II 1934) vom Wyler Berg bei Kleve (80\% Pinus, Picea und Abies, $12 \%$ Alnus; später von G. von DER BRELIE \& U. ReIN 1952 als Bruchwaldtyp eines kälteren Abschnittes des Mindel/Riß-Interglazials gedeutet), U. REIN (1950 und besonders in: H. KarReNBerg \& U. REIN 1951) vom Hülser Berg bei Krefeld (6 Proben, $5-25 \%$ Abies) und G. vON DER BRELIE und U. REIN (1952) vom Tönisberg, Dachsberg und Wyler Berg (in allen Proben geringe Abies-, dafür hohe Alnus- und Pinus-Werte, deshalb als Bruchwaldtypen eines kälteren Abschnittes des Mindel/Riß-Interglazials gedeutet; vgl. Abb. 5).

10) Als primäre Lagerstätte kann die den Stauchwällen östlich vorgelagerte Basisfläche der Unteren Mittelterrasse des Rheines zwischen Krefeld und Kleve gelten. lesen.

11) Auf der geologischen Spezialkarte 1:25000 (Bl. Crefeld) ist Haideck statt Heideck zu 


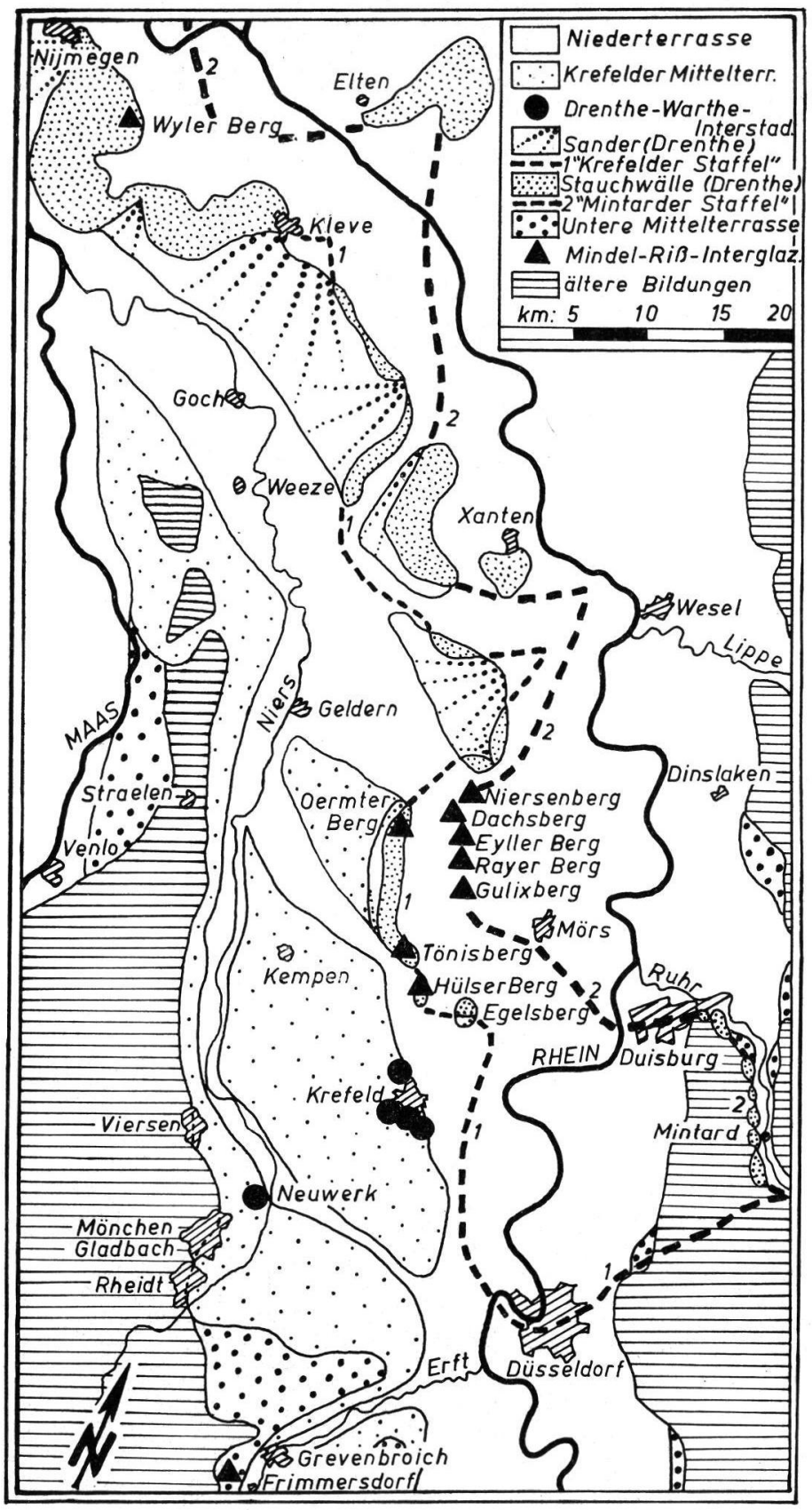

Abb. 5. Die Verbreitung mittelpleistozäner Ablagerungen in der Niederrheinischen Bucht zwischen Dïsseldorf und Nijmegen.

(Nach H. W. Quitzow 1956, J. I. S. Zonneveld 1956, K. Kaiser 1957, K. N. Thome 1958, 1960 u. a.) 
bückerhof und der St. Töniser Straße bei Krefeld und U. REIN (H. Karrenberg \& U. REIN 1951) vom Wasserwerk 1, Großbückerhof und der St. Töniser Straße bei Krefeld durchgeführten pollenanalytischen Untersuchungen haben auf Grund hoher TannenWerte, aber wenig Eichenmischwald-, Hasel- und Fichten-Pollen weitgehende Übereinstimmung mit den Krefelder Schichten gezeigt.

Ist es aber berechtigt, die von A. Steeger (K. Bertsch, A. Steeger \& U. Steusloff 1931) als Kempener Schichten bezeichneten fossilführenden Ablagerungen der Krefelder Mittelterrasse den Krefelder Schichten gleichzustellen, wie das zunächst H. KarRENBERG und U. REIN (1951) - und seitdem fast alle sonstigen Autoren - getan haben ${ }^{12}$ )? Nicht nur aus faunistischen, sondern auch aus stratigraphischen Gründen erheben sich gegen diese Gleichsetzung jedoch Bedenken. Die in den Stauchwällen die Krefelder Schichten ummantelnden Sand-Kies-Folgen müssen älter sein als der rißhochglaziale Eisvorstoß (Drenthe-Stadium oder Saale-Eiszeit i.e.S.) in die Niederrheinische Bucht. Darin können somit Serien enthalten sein, die wohl größtenteils der Unteren Mittelterrasse des Rheines, z. Ti. aber wohl auch noch älteren Ablagerungen angehören, wie u. a. durch sedimentpetrographische Untersuchungen durch J. D. DE Jong (1956) und G. C. MAarleveld (1956) erwiesen werden konnte. Demgegenüber besitzen die hangenden Sand-Kies-Folgen (Krefelder Mittelterrasse) über den Kempener Schichten ganz oder größtenteils ein jüngeres Alter als jener Eisvorstoß in die Niederrheinische Bucht. Die Ablagerungen der Krefelder Mittelterrasse sind mit größter Wahrscheinlichkeit dem Warthe-Stadium der RißEiszeit (Saale-Eiszeit i.w.S.) zuzuordnen (vgl. u. a. H. W. Quitzow 1956, K. KAISER 1957). Die liegenden Sand-Kies-Folgen der Kempener Schichten sind in Ubereinstimmung mit H. W. Quirzow (1956) dem „Rinnenschotter“ (Mittlere Mittelterrasse des Rheines) gleichzustellen ${ }^{13}$ ), nachdem J. D. DE JoNG (1956) sedimentpetrographisch nachgewiesen hat, daß diese nicht als Reste der rheinischen Hauptterrasse, wie es vordem H. KarRenberg und U. REIN (1951) angenommen hatten, gedeutet werden können. Ob sich schließlich noch im unmittelbaren Hangenden oder Liegenden der Kempener Schichten auch Reste

12) A. SteEger selbst (1952) hat betont, daß die Übereinstimmung in der Flora zwar groß ist, daß sich andererseits aber auch in den letztinterglazialen "Mörser Schichten" durch K. JesseN (1934, vgl. A. STEEger 1952) eine Zone hat nachweisen lassen, die den Krefelder Schichten hinsichtlich ihres Pollengehaltes ebenfalls sehr ähnlich ist. Außerdem hat U. STEUSLOFF (K, BERTSCH, A. StEeger \& U. Steusloff 1931) auf die Verschiedenheiten der Faunen der Krefelder Schichten gegenüber den Kempener Schichten hingewiesen. So haben vor allem P. G. Krause (1909), G. Fliegel (G. Fliegel \& J. Stoller 1910) und A. Steeger (1923, K. Bertsch \& A. Steegér 1927) von zahlreichen Fundpunkten der Krefelder Schichten (Hülser Berg, Tönisberg, Oermter Berg, Gulixberg, Rayer Berg, Eyeller Berg, Dachsberg, Niersenberg, Wyler Berg, vgl. Abb. 5) eine Muschel- und Schneckenfauna bekannt gemacht - mit Viviparus (=Paludina) diluvianus (KuNTH) und Valvata naticina MENKE -, die der des "Neediens" der Niederlande (von Neede und Herikerberg) und der "Paludinen-Schichten" Nordwestdeutschlands (vgl. u. a. P. WolDstedt 1955) übereinstimmt. Demgegenüber sind die Kempener Schichten nach U. Steusloff (K. BerTsCh, A. Steeger \& U. Steusloff 1931) durch eine Mollusken-Gemeinschaft gekennzeichnet, wie sie in jüngster Zeit noch die Auenwälder des Oberrheines besiedelt hat.

13) Nach G. C. MaArLeveld (1956) sind geringe Unterschiede (im Quarzgehalt) der Geröllzusammensetzung der liegenden gegenüber den hangenden Sand-Kies-Folgen der Kempener Schichten unverkennbar. Entgegen der Ansicht von J. D. DE JoNG (1956), wonach beide Folgen hinsichtlich der Schwermineralverteilung weitgehende Ubereinstimmung zeigen sollen, möchte ich mit H. W. Quitzow (1956) annehmen, daß sich die Liegendserien durch einen höheren und daher für die "Rinnenschotter" typischen Gehalt an vulkanischen Mineralen (Augit, Hypersthen, Basaltische Hornblende, Titanit) gegenüber den Hangendserien auszeichnen. Allerdings kann ich mich der Ansicht von H. W. QuITzow (1956) nicht anschließen, daß die „Rinnenschotter“ stratigraphisch dem Mindel/Riß-Interglazial zuzuordnen seien. Sie dürften vielmehr in das Ende der Mindel-Eiszeit zu stellen sein (vgl. auch P. Woldstedt 1958 und G. von DER BreLIE, K. KilpPER \& R. TeIChmüller 1960). Auch sollte der Begriff „Rinnenschotter" durch „Ablagerungen der Mittleren Mittelterrasse des Rheines" für das Niederrheingebiet ersetzt werden, worauf ich zuvor schon eingegangen bin. 
der Unteren Mittelterrasse verbergen, kann nicht mit Sicherheit entschieden werden ${ }^{14}$ ). Die in situ erschlossenen oder erbohrten Krefelder Schichten (Frimmersdorf an der Erft, Wasserwerk Bensberg, Kiesgrube bei Efferen?) sind über $2 \mathrm{~m}$ mächtig und wurden übereinstimmend in einem Niveau 15-16 m unter der Oberkante der Unteren Mittelterrasse des Rheines angetroffen. Demgegenüber sind die Kempener Schichten nur weniger als $1 \mathrm{~m}$ mächtig und in einer Tiefenlage von 12-13 m unter der Oberkante der Krefelder Mittelterrasse erbohrt worden. Wenn man die Unterschiede der Mächtigkeiten außer Acht läßt und andererseits bedenkt, daß die Krefelder Mittelterrasse nur einige Meter in die Untere Mittelterrasse eingeschachtelt ist, so kamen demnach beide fossilführende Schichtenserien im gleichen Niveau zur Ablagerung. Trotzdem ist aber auf Grund stratigraphischer Erwägungen der Schluß auf eine rißinterstadiale Stellung (Drenthe/Warthe-Interstadial) der Kempener Schichten gegenüber der mindel/riß-interglazialen Stellung der Krefelder Schichten weit zwingender. Nach den sedimentpetrographischen Befunden (vgl. Anm. 14) ist es (wie zuvor schon angedeutet) unwahrscheinlich, daß sich im unmittelbaren Hangenden der Kempener Schichten noch Reste der Unteren Mittelterrasse befinden. Allerdings wäre ja auch dieser Nachweis schwer zu führen (vgl. Anm. 14). Solange wir also nicht in entsprechend tiefen Aufschlüssen des Krefelder Raumes eine Erosionsdiskordanz innerhalb der Schotterserien über den Kempener Schichten feststellen oder den sicheren Nachweis der sedimentpetrographischen Zugehörigkeit von Sand-Kiєs-Folgen unmittelbar über den Kempener Schichten zur Unteren Mittelterrasse führen können, ist es nach den z. Zt. vorliegenden Untersuchungsbefunden weit wahrscheinlicher anzunehmen, daß die gesamten Hangendserien der Kempener Schichten einer einheitlichen Akkumulationsfolge zugehören, daß also nach einer gewaltigen Erosion vor Ausbildung der Mittleren Mittelterrasse und nach einer weniger tiefen Erosion vor Ausbildung der Unteren Mittelterrasse schließlich nochmal eine Erosion mit gleicher Tiefenwirkung als zuvor erfolgte, die kurz nach dem Eisvorstoß in die Niederrheinische Bucht begann und erst am Ende des nachfolgenden Interstadials durch eine erneute Akkumulation (Krefelder Mittelterrasse) abgejöst worden sein dürfte. Es wurde ferner zuvor schon angedeutet, daß nicht nur die bisher vorliegenden stratigraphischen Untersuchungsbefunde, sondern in noch weit höherem Maße die Faunenunterschiede eine zeitliche Trennung der Kempener von den Krefelder Schichten anzeigen (vgl. Anm. 12).

Sprechen aber die floristischen Befunde vollkommen gegen eine solche Deutung? Nachdem H. Illies $(1952,1955)$ die stratigraphischen Vorarbeiten geleistet hatte, zögerte G. von DER Brelie (1955) nicht, die palynologisch von R. GistL (1928), U. Rein (in: P. Woldstedt 1949) und W. Selle (1954 und in: P. Woldstedt, U. Rein \& W. Selle 1951) untersuchten Kieselgurvorkommen von Ober- und Neu-Ohe, sowie von Munster in der Lüneburger Heide als „Ohe-Interglazial“ dem Drenthe/Warthe-Interstadial zuzuordnen, wobei er allerdings auf die durch die Pollenspektren angezeigte große Übereinstimmung hinsichtlich des Vegetationsablaufes zum Mindel/Riß-Interglazial hinweist. Es zeigt sich nun, daß die Kempener Schichten floristisch ohne Bedenken der Zone VIII (Erlen-Kiefern-T a n n e n-Zeit) und vielleicht auch z. T. der Zone IX (Erlen-K i e f e r nZeit) des „Ohe-Interglazials“ gleichgestellt werden können (vgl. H. KARRENBERG \& U.

14) Nach J. I. S. ZonNEveld (1956) unterscheiden sich Untere Mittelterrasse, Krefelder Mittelterrasse und (Altere) Niederterrasse des Rheines in der Niederrheinischen Bucht hinsichtlich ihrer Schwermineralverteilung kaum voneinander. Lediglich soll sich die Krefelder Mittelterrasse durch einen etwas größeren Gehalt an Basaltischen Hornblenden gegenüber den beiden anderen Terrassen auszeichnen, was sich aber durch weiträumigere Untersuchungen nicht bestätigt hat. Leider ist auch aus den Schwermineraldiagrammen von J. D. DE Jong (1956) des Kempener Feldes die Verteilung der Basaltischen Hornblende nicht zu ersehen. M. E. scheint ein Kriterium zur Unterscheidung beider Terrassen darin gegeben zu sein, daß der Augitgehalt der Unteren Mittelterrasse durchschnittlich 5\% höher ist als in der Krefelder Mittelterrasse. 
REIN 1951, G. von DER Brelie 1955) ${ }^{15}$ ). Andererseits zeigen die Neuwerker Schichten ${ }^{16}$ ), die nach stratigraphischen Erwägungen gleich den Kempener Schichten demselben Interstadial zugeordnet werden müßten, eine geringe Übereinstimmung zu den Zonen VI/VII (2. E i che $\mathrm{nm}$ i s ch w a ld-Hasel-Zeit/H a i $\mathrm{buchen-Zeit)} \mathrm{des} \mathrm{"Ohe-Interglazials"}$ (vgl. K. Bertsch, A. Steeger \& U. Steusloff 1931, G. von der Brelie 1955); allerdings ist diese Zuordnung auch wieder palynologisch durch die überaus hohen HainbuchenWerte (z. T. über 70\%) und vor allem durch das Auftreten der Buche (bis 20\%; wahrscheinlich liegt eine Fehlbestimmung vor, denn Buche ist bisher in diesem Maße nur aus dem Postglazial bekannt) stark in Frage gestellt. P. Woldstedt (u. a. 1958) hat jedoch berechtigte Zweifel hinsichtlich der stratigraphischen Stellung des „Ohe-Interglazials“ und zugleich auch des von E. Kolumbe (1953) beschriebenen Hemmor-Torfes (vgl. auch G. voN DER BRELIE 1955) als drenthe/warthe-interstadiale Bildungen erhoben, so daß ein Vergleich der Floren der Kempener und Neuwerker Schichten mit den zuvor genannten vorerst noch nicht, vor allem, was die stratigraphischen Ausdeutungen anbelangt, berechtigt erscheint. W. SzAFER (1957) hat die wichtigsten und vollständigsten Pollendiagramme des Drenthe/Warthe-Interstadials im mittel- und osteuropäischen Raume zusammengestellt, wobei er allerdings das „Ohe-Interglazial“ hinzurechnete. Dabei kann als gesichert gelten, daß die Diagramme von Brzozowica (Stuchlik \& RALSKa 1957), Labedy (RALSKA 1957) in Polen und Suraz (Czebotariewa 1953) in der Sowjet-Union diesem Interstadial zugehören. Es zeigt sich nun aber auch, daß ein floristischer Vergleich der Kempener und Neuwerker Schichten zu den zuvor erwähnten Interstadialen Polens und der SowjetUnion gar nicht abwegig ist. So stimmen die Kempener Schichten gut mit oberen Abschnitten jener Interstadiale überein, wenn man davon absieht, daß Tanne umgekehrt häufig hier und dort erscheinen. Andererseits ist aber auch die Hainbuche in mittleren Abschnitten der polnischen Interstadiale (besonders Labedy) derart häufig, daß ebenfalls eine gewisse Übereinstimmung der Neuwerker Schichten mit mittleren Abschnitten der polnischen Interstadiale (abgesehen von Buche!) besteht. Auf jeden Fall würden somit die Floren nicht unbedingt gegen eine Abtrennung der Kempener und Neuwerker Schichten von den mindel/riß-interglazialen Krefelder Schichten sprechen, nachdem zuvor schon dargelegt wurde, daß diese Abtrennung auf Grund der Faunenverschiedenheit und stratigraphischer Erwägungen gefordert werden muß ${ }^{17}$ ).

15) Man kann sich der Anschauung von U. REIN (1955) nicht anschließen, wonach sich Krefelder und Kempener Schichten hinsichtlich ihrer Pollenführung von denen des "Ohe-Interglazials" unterscheiden sollen. Die Berufung hinsichtlich dieser Feststellung auf G. voN DER BRELIE (1955) ist nicht recht verständlich, da letzterer keine floristischen Vergleiche der Krefelder und Kempener Schichten zu denen des „Ohe-Interglazials“ durchgeführt hat. Im Gegenteil ist besonders von G. von Der Brelie (1955) auf die große Ähnlichkeit der Pollenführung beider Interglaziale hingewiesen worden. Es kann daher nur noch einmal mit Nachdruck betont werden, daß die Gründe für die Datierung des "Ohe-Interglazials" in das Drenthe/Warthe-Interstadial (Gerdau-Interstadial) in erster Linie stratigraphischer Natur waren, wogegen dann allerdings wieder berechtigte Zweifel erhoben worden sind (vgl. u. a. P. WolDSTEDT 1958).

16) Das basale Tonlager mit pflanzenführenden Torfen in der Krefelder Mittelterrasse der Bohrung Neuwerk bei Mönchen Gladbach hat A. STEEger (1952) als Neuwerker Schichten bezeichnet, nachdem er es zuvor (in: K. Bertsch, A. SteEger \& U. Steusloff 1931) den Kempener Schichten gleichgestellt hatte.

17) Aber nicht nur die Kempener und Neuwerker Schichten dürften innerhalb der Niederrheinischen Bucht einem Interstadial zuzuordnen sein. Ein gleiches gilt mit größter Wahrscheinlichkeit auch für das von G. von DER BRELIE, A. Mückenhausen \& U. Rein (1956) aus den Niederterrassen-Sedimenten von Weeze beschriebene Torflager. Entgegen der Meinung der Bearbeiter kann man in Ubereinstimmung mit U. Jux \& K. A. Rosenbauer (1959) die Florenfolge von Weeze mit der von H. Nietsch (1940) aus einem alten Rheinarm bei Köln-Merheim beschriebenen spät- bis postglazialen Alters zur Deckung bringen, woraus letztere den Schluß zogen, daß diese auch altersgleich wären. Sie haben jedoch nicht in Betracht gezogen, daß die fossilführenden Schichten von Weeze von ca. $4 \mathrm{~m}$ mächtigen Schottern überlagert werden, die wegen der Bimsfreiheit mit größter Wahrscheinlichkeit der Alteren Niederterrasse zugeordnet werden müssen. Insofern be- 
G. von der Brelie, K. Kilpper \& R. Teichmüller (1960) haben schließlich von Frimmersdorf an der Erft ein Pleistozänprofil beschrieben (Abb. 5), wo erstmalig neben einem "Interstadial"18) auch ein Interglazial in situ aufgeschlossen vorgefunden wurde, das nach stratigraphischen Erwägungen (R. TEICHMÜLLER) ohne Zweifel dem Mindel/RißInterglazial zuzuordnen ist. Sowohl die mikro- (G. vON DER BRELIE) als auch die makrofloristischen Befunde ( $\mathrm{K}, \mathrm{K}$ ILPPER) ergeben allerdings ein stark abweichendes Bild von den Standardprofilen dieses Interglazials (Abies wurde nur makrofloristisch nachgewiesen; Juglans, Pterocarya und Keteleeria-ähnliche Arten in einem Erlen-Kiefernwald!) ${ }^{19}$ ).

Die zuvor ermittelten Ergebnisse seien in nachfolgender Tabelle zusammengefaßt:

steht also hinsichtlich der Lagerungsverhältnisse ein Unterschied zu denen von Köln-Merheim. Andererseits werden aber auch bei Weeze die Torfe von Terrassen-Sedimenten unterlagert, die G. von DER Brelie, A. MüCKenhausen \& U. Rein (1956) ohne sedimentpetrographische Úberprüfung der "Mittelterrasse" (es dürfte der "Rinnenschotter" gemeint sein) zuordneten. In der Kiesgrube der Wtw. Boll \& Sohn in Weeze-Kalbeck (unweit der Fundstelle an der Weezer Kirche) ist ein ähnliches Schichtenprofil aufgeschlossen. Unter Auelehmen $(1 \mathrm{~m})$ und ca. $4 \mathrm{~m}$ mächtigen Sand-Kies-Folgen mit auffälliger basaler Grobblockzone und ohne Bimssteinlagen finden sich in einer Tiefe von 5-6 m grau-braune Tone mit einem Torflager. Aus dem Ton unmittelbar über den Torfen konnten zahlreiche Holzreste (Prof. H. Werland, Wuppertal, bestimmte u. a.: Carpinus betulus, Pinus silvestris), Reste von Säugetieren (u. a.: Zähne und Geweihteile von Cervus elaphus LinN., Zähne von Bos primigenius Boj. und auch in Geoden verbackene und umgelagerte tertiäre Meeresmuscheln (Oberoligozän) geborgen werden. Das Profil wird nach unten mit Terrassensedimenten gleicher Zusammensetzung wie die hangenden Sand-Kies-Folgen abgeschlossen, die deshalb auch wohl nicht der Mittleren Mittelterrasse, sondern der Alteren Niederterrasse zuzuordnen sind, über Blautonen (beginnend in 7,50 m Tiefe), die vermutlich den Tegelen-Schichten gleichzustellen sind. Eine Holzprobe (Carpinus betulus) wurde im C14-Labor des 2. Phys. Inst. der Universität Heidelberg durch Dr. K. O. MünNICH, dem herzlich gedankt sei, unter der Nr. H 405437 „Holz vom Forsthaus Sandheide bei Weeze" untersucht, wobei ein Alter von „älter als 35000 Jahren“ ermittelt wurde. Daraus kann aber nur der Schluß gezogen werden, daß auch die fossilführenden Ablagerungen nicht einem Interglazial (Eem-Interglazial), sondern einem relativ lang dauerndem und warmen Interstadial (vermutlich einem frühen Würm-Interstadial [?Göttweig?] zuzuordnen sind.

18) Dieses wird als Riß I/II-Interstadial einzustufen versucht, worunter man an sich das Gerdau-Interstadial (vgl. u. a. G. LÜTrIG 1958) verstehen würde. Da es sich aber hierbei um Schichten handelt, die basalen Lagen der Unteren Mittelterrasse (Drenthe-Stadium) eingelagert sind, also älter als jenes Interstadial sein müssen, so wäre es wohl richtiger, dieses einem DrentheIntervall zuzuordnen. Einerseits wissen wir zur Zeit noch nicht, ob im Frühriß, z. B. etwa zwischen der Rehburger Zeit und dem Drenthe-Abschnitt (i.e.S.) überhaupt ein echtes Interstadial existiert hat, und andererseiten deutet die Florengemeinschaft des Frimmersdorfer "Interstadials" im Hinblick auf Dauer und Klima wohl eher auf ein Intervall hin. Sinngemäß würden hier aber wohl auch die gleichen Bemerkungen zur Nomenklatur der Gliederung der Riß-Eiszeit Gültigkeit besitzen, die beispielsweise vor allem P. WolDSTEDT (1955) für die der Würmeiszeit geltend zu machen versuchte.

19) Die Flora und in noch weit höherem Maße die Käfer-Fauna weisen darauf hin, daß jene fossilführenden Schichten von Frimmersdorf an der Erft an das Ende (zumindest in einen relativ kühlen Abschnitt) des Mindel/Riß-Interglazials zu stellen sind. Um so mehr muß es daher verwundern, daß in einem Wald, in dem Kiefer und Erle dominieren, hin und wieder äußerst wärmeliebende Arten (Pollen und Nüsse von Juglans und Pterocarya, Keteleeria-ähnliche Pollen und Zapfen) auftreten sollen, was als sehr fraglich angesehen werden muß. Die Gründe, die für eine Autochthonie dieser äußerst wärmeliebenden Komponenten angegeben werden, sind m. E. nicht unbedingt zwingend, denn:

1) das Fehlen sonstiger wärmeliebender Floren (Tertiärrelikte) besonders in den Pollen-Spektren spricht nicht unbedingt gegen eine Allochthonie der vorgefundenen Elemente,

2) das Vorkommen von Pterocarya-Nüssen und Keteleeria-verdächtigen Zapfen-Resten muß auch nicht notwendig auf Autochthonie hinweisen, selbst wenn in den liegenden Sanden bislang diese nicht gefunden wurden und auch in Flöz Frimmersdorf der benachbarten Grubenbezirke bisher keine Pterocarya-Nüsse beobachtet wurden. Gerade das reiche Auftreten verschwemmter Tertiärpollen in den liegenden Sanden sollte zur Vorsicht gemahnen, die tertiären Relikte unter den Florenresten der interglazialen Schichten als autochthon hinzustellen. Auch in StillwasserBereichen oder in wenig bewegtem Wasser sind $\mathrm{m}$. E. Umlagerungen von Florenresten durchaus möglich. Man sollte ferner bedenken, daß hierbei Korrodierungen von Mikro- und Makrofloren- 
Tabelle 2

Chronologische Gliederung mittel- und jungpleistozäner Schichten und deren Klimaindikatoren in der Niederrheinischen Bucht.

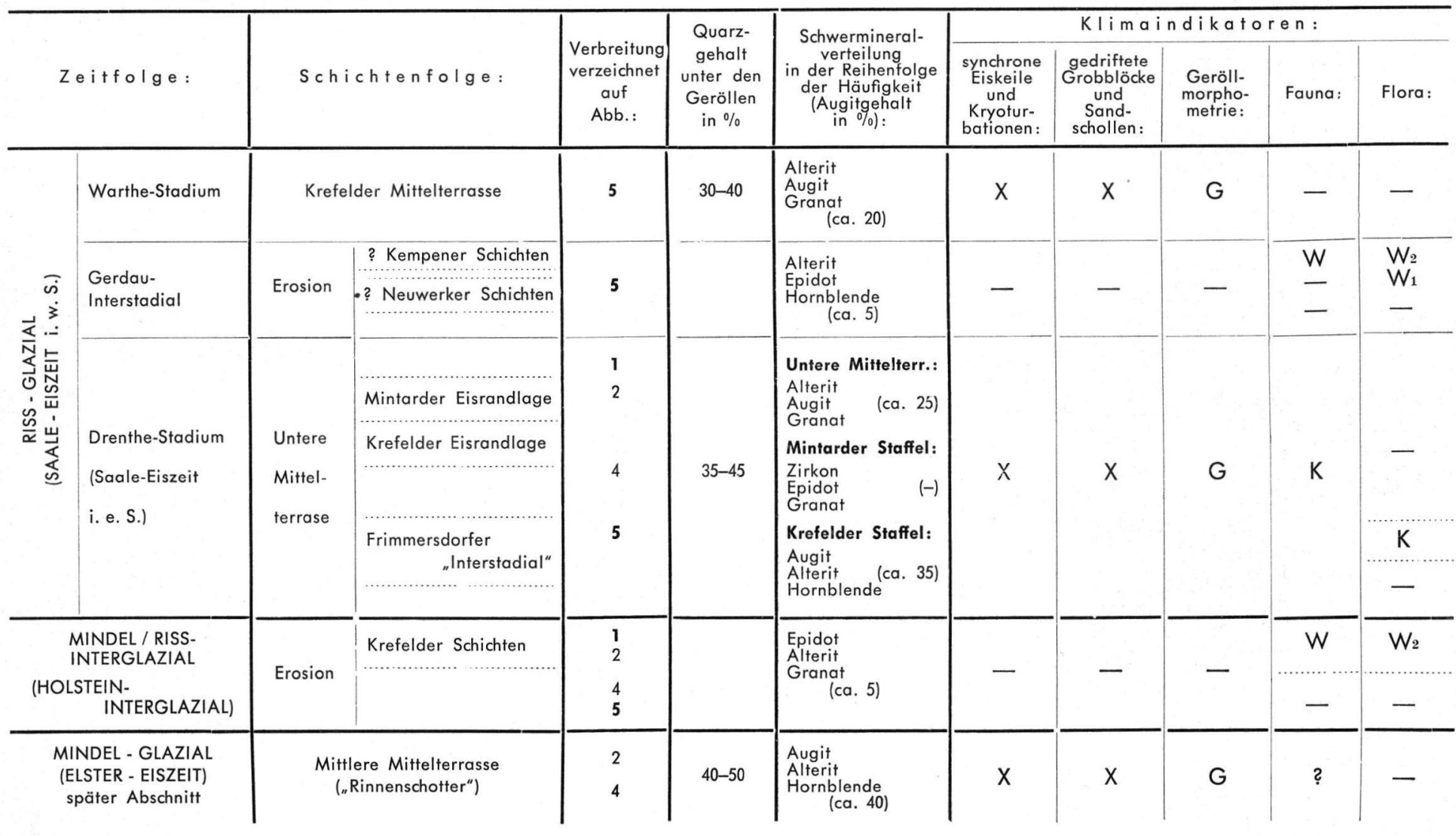

$(\mathrm{X}=$ vorhanden; $?=$ vorhanden, aber nicht klimatisch ausdeutbar; $-=$ nicht vorhanden; $\mathrm{G}=$ deutet auf glaziale Klimabedingungen hin; $\mathrm{K}=\mathrm{kaltzeitliche} \mathrm{[Säuger-]}$ Fauna oder Flora; W = warmzeitliche [Mollusken-] Fauna oder Flora $\left[W_{1}=\right.$ deutet auf optimales Warmklima hin, W $=$ deutet auf gemäßigteres Warmklima hin]). 
resten äußerst selten und von weit geringem Grad als bei stärker bewegtem Wasser erfolgen. Vielleicht erlauben auch die jetzt durch Prof. MurawskI am geologischen Institut Köln in Angriff genommenen gründlichen Untersuchungen der Käfer-Fauna nähere Aussagen hinsichtlich der klimatischen und zeitlichen Situation zu machen. Desgleichen würden verfeinerte sedimentpetrographische Untersuchungen und eine Erweiterung der vergleichenden Untersuchung über den Floreningehalt von Flöz Frimmersdorf in den benachbarten Grubenbezirken wesentlich zur Klärung dieser Fragen beitragen.

\section{Schriftenverzeichnis}

Braun, F. J.: Die Terrassengliederung am linken Niederrhein zwischen Geldern und Kalkar. Geol. en Mijnb., N. S. 18, 374-378, 's-Gravenhage 1956.

Brelie, G. voN DER: Die pollenstratigraphische Gliederung des Pleistozäns in Nordwestdeutschland. 2. Die Pollenstratigraphie im jüngeren Pleistozän. - Eiszeitalter u. Gegenwart 6, 25-38, O'hringen/Württ. 1955.

Brelie, G. voN DER, KilpPeR, K. \& TeichmülleR, R.: Das Pleistozän-Profil von Frimmersdorf an der Erft. - Fortschr. Geol. Rheinld. u. Westf. 4, 179-196, Krefeld 1960.

Brelie, G. von Der, Mückenhausen, A. \& Rein, U.: Ein Torf aus dem Eiszeitalter im Untergrund von Weeze. - Der Niederrhein 22, 1-4, Krefeld 1955.

Brelie, G. von DER \& REIN, U.: Die Interglazialbildungen im Niederrheinischen Diluvium. Der Niederrhein 19, 63-68, Krefeld 1952. - - Pollenanalytische Untersuchungen zur Gliederung des Pleistozäns am linken Niederrhein. - Geol. en Mijnb., N. S. 18, 423-425, 's-Gravenhage 1956.

Brelie, G. von Der, Rein, U., Klusemann, H., Teichmüller, R. \& Wortmann, H.: PleistozänProfile im Essener Raum. - N. Jb. Geol. Paläont., Mh. 1956, 113-132, Stuttgart 1957.

Hopmann, M., Frechen, J. \& KnFtsch, G.: Die vulkanische Eifel. - 2. Aufl., 1-144, Bonn 1958.

IlliEs, H.: Eisrandlagen und eiszeitliche Entwässerung in der Umgebung von Bremen. - Abh. nat. Ver. Bremen 33, 19-56, 1952. - - Die Vereisungsgrenzen in der weiteren Umgebung Hamburgs, ihre Kartierung und stratigraphische Bewertung, - Mitt. geogr. Ges. Hamburg 51, 7-54, 1955.

JoNG, J. D. DE: Sedimentpetrographische Untersuchungen in Terrassenschottern im Gebiet zwischen Krefeld und Kleve. - Geol. en Mijnb., N. S. 18, 389-394, 's-Gravenhage 1956.

Jux, U. \& RosenbaueR, K. A.: Zum Vorkommen von Cetaceen-Resten in jungpleistozänen Flußablagerungen der Niederrheinischen Bucht. - N. Jb. Geol. Paläont., Abh. 108, 81-126, Stuttgart 1959.

KAISER, K.: Geologische Untersuchungen über die Hauptterrasse in der Niederrheinischen Bucht. Sonderveröff. Geol. Inst. Köln 1, 1-68, Köln 1956. - - Die Höhenterrassen der Bergischen Randhöhen und die Eisrandbildungen an der Ruhr. - Ebendort 2, 1-39, Köln 1957. - - Wirkungen des pleistozänen Bodenfrostes in den Sedimenten der Niederrheinischen Bucht. Ein Beitrag zur Kenntnis der Periglazialerscheinungen der Rheinlande. - Eiszeitalter u. Gegenwart 9, 110-129, OOhringen/Württ. 1958.

Karrenberg, H.: Verbreitung und Lagerung von Haupt- und Mittelterrasse am Niederrhein. Z. deutsch. geol. Ges. 103, 118, Hannover 1951.

Lürtig, G.: Eiszeit-Stadium-Phase-Staffel. Eine nomenklatorische Betrachtung. - Geol. Jb. 76, 235-260, Hannover 1958.

MaArleveld, G. C.: Ergebnisse von Kiesanalysen im Niederrheingebiet. - Geol. en Mijnb., N. S. 18, 411-415, 's-Gravenhage 1956.

Quitzow, H. W.: Die Terrassengliederung im niederrheinischen Tieflande. - Ebendort 18, 357-373, 's-Gravenhage 1956.

REIN, U.: Über Interglazialbildungen im niederrheinischen Diluvium. - Z. deutsch. geol. Ges. 103, 117-118, Hannover 1951. - - Die pollenstratigraphische Gliederung des Pleistozäns in Nordwestdeutschland. 1. Pollenstratigraphie im älteren Pleistozän. - Eiszeitalter u. Gegenwart 6, 16-24, OOhringen/Württ. 1955.

Schwarzbach, M.: Das Klima der Vorzeit. - Stuttgart 1950.

SteEger, A.: Das glaciale Diluvium des niederrheinischen Tieflandes. Beitrag I-VII. - Ber. niederrh. geol. Ver. 1923, C 1-46, Bonn 1925 (mit einem Literaturverzeichnis bis 1923). - 100 Jahre Eiszeitforschung am Niederrhein. - Der Niederrhein 19, 55-63 u. 86-87, Krefeld 1952 (mit einem Literaturverzeichnis bis 1952).

SZAFER, W.: Úber die Zweiteilung des Riß-Glazials. - Veröff. geobot. Inst. Rübel in Zürich 34, 126-131, Bern u. Stuttgart 1957.

Tноме, K. N.: Die Begegnung des nordischen Inlandeises mit dem Rhein. - Geol. Jb. 76, 261-308, Hannover 1958. - - Eisvorstoß und Flußregime an Niederrhein und Zuider See im Jungpleistozän. - Fortschr. Geol. Rheinld. u. Westf. 4, 197-246, Krefeld 1960. 
VLERK, I. M. VAN DER \& FLoRschüTZ, F.: Nederland in het Ijstijdvak. - Utrecht 1950 (mit einem Literaturverzeichnis für die Niederlande). - - The palaeontological base of the subdivision of the Pleistocene in the Netherlands. - Verh. kon. ned. Akad. Wetensch., Afd. Natuurk., 1. Reihe 20, 1-58, Amsterdam 1953.

Woldstedt, P.: Saaleeiszeit, Warthestadium und Weichseleiszeit in Norddeutschland. - Eiszeitalter u. Gegenw. 4/5, 34-48, Öhringen/Württ. 1954. - - Norddeutschland und angrenzende Gebiete im Eiszeitalter. - 2. Aufl., Stuttgart 1955. - - Úber die Gliederung der Würm-Eiszeit und die Stellung der Lösse in ihr. - Eiszeitalter u. Gegenw. 7, 78-86, Óhringen/Württ. 1955. - - Das Eiszeitalter. Grundlagen einer Geologie des Quartärs. 2. Aufl., Stuttgart 1954 u. 1958.

Zonneveld, J. I. S., Schwermineralgesellschaften in niederrheinischen Terrassensedimenten. - Geol. en Mijnb., N. S. 18, 395-401, 's-Gragenhage 1956. - - Litho-stratigraphische eenheden in het Nederlandse Pleistoceen. - Meded. geol. Sticht., N. S. 12, 31-64, Maastricht 1958.

Manuskr. eingeg. 20. 8. 1960.

Anschrift der Verfasser: Dr. Karlheinz Kaiser, Geol. Inst. der Universität, Köln, Zülpicher Str. 47; Dr. Rudolf Schütrumpf, Inst. für Ur- und Frühgeschichte der Universität, KölnLindenthal, Weyertal 125. 